\title{
3 Research Square

\section{Grooved Pegboard Test Performance Before and After Cerebral-Spinal Fluid Tap Test in Patients with Normal Pressure Hydrocephalus}

\section{Caiyan Liu}

Peking Union Medical College Hospital https://orcid.org/0000-0001-6035-7456

\section{Liling Dong}

Peking Union Medical College Hospital

\section{Chenhui Mao}

Peking Union Medical College Hospital

Jie Li

Peking Union Medical College Hospital

\section{Xinying Huang}

Peking Union Medical College Hospital

\section{Junji Wei}

Peking Union Medical College Hospital

\section{Bo Hou}

Peking Union Medical College Hospital

\section{Feng Feng}

Peking Union Medical College Hospital

\section{Liying Cui}

Peking Union Medical College Hospital Jing Gao ( $\sim$ gj107@163.com )

\section{Research}

Keywords: Normal pressure hydrocephalus, cerebral-spinal fluid tap test, Grooved pegboard test

Posted Date: May 19th, 2020

DOI: https://doi.org/10.21203/rs.3.rs-29451/v1

License: (c) (i) This work is licensed under a Creative Commons Attribution 4.0 International License.

Read Full License 


\section{Abstract}

Background Motor impairment in patients with normal pressure hydrocephalus (NPH) can extend beyond gait and include deficits in upper extremity functions and psychomotor speed. Evaluation of upper extremity function will be helpful for NPH patients who are unable to ambulate (e.g., wheelchair-bound patients) and may not be able to comply with the gait evaluation. Our study aimed to explore the use of the grooved pegboard test to assess responsiveness to the cerebrospinal fluid (CSF) tap test in patients with NPH.

Methods Seventy-seven possible NPH patients were enrolled from 2013 to 2018 . All patients underwent detailed neuropsychological and walking assessments, CSF tap tests, and brain magnetic resonance imaging. The grooved pegboard test results before and after the CSF tap test were compared and correlated with the other clinical assessments. In diffusion tensor imaging analysis, the fractional anisotropy (FA) and mean diffusivity (MD) values of periventricular white matter were measured by the region of interest method and were correlated with pegboard test performance.

Results The grooved pegboard test scores significantly improved after the CSF tap test and correlated with patient walking ability, cognitive function, and functional scores $(P<0.01)$. The improvement ratios in the complex visual motor speed index (i.e., the grooved pegboard test performance combined with the Symbol-Digit Modalities Test performance) were significantly different between the CSF tap test responder and nonresponder groups. The grooved pegboard test times were significantly correlated with the FA values in right periventricular lesions $(P=0.017)$.

Conclusions The performance on the grooved pegboard test was related to lower extremity motor ability and cognitive function. It can be used as an alternative evaluation tool for patients who are unable to ambulate and may not be able to comply with the gait evaluation.

\section{Background}

Normal pressure hydrocephalus (NPH) patients present disturbances in gait and balance, cognition and/or control of urination with neuroimaging characterized by enlargement of the cerebral ventricles [13]. Typically, gait disturbance in NPH patients is the initial and most prominent symptom [4, 5], but motor impairments can also extend to include upper extremity dysfunction [6-9]. Cognitive impairment in NPH patients is generally characterized by frontal system deficits that may include slowing of information processing, task shifting, reduced learning and executive abilities [10-12]. Bladder control dysfunction can range from increased urinary urgency and frequency to frank incontinence.

A commonly used auxiliary test for predicting shunt responsiveness is the cerebrospinal fluid (CSF) tap test (TT), a procedure in which NPH symptoms are assessed before and after drainage of $30 \sim 50 \mathrm{~cm} 3$ of CSF by lumbar puncture. Clinical improvement following lumbar puncture indicates an increased likelihood of improvement after shunt placement [13-15]; however, a negative CSF TT does not rule out shunt responsiveness [16]. Standard methods for determining the CSF TT response are based on the 
clinical impression of changes in gait and cognition after lumber puncture. Instruments to evaluate gait $[17,18]$ can be useful in this assessment but are not always sensitive to subtle changes. NPH patients who are unable to ambulate (e.g., the patient is wheelchair bound) may not be able to comply with the gait evaluation but still benefit from shunt placement. In mildly affected patients, testing may not reveal sufficiently profound impairments to permit assessment of change after a lumber puncture. Therefore, we sought to identify additional measures that can be used to objectively assess responsiveness to the CSF TT.

The grooved pegboard test assesses eye-hand coordination and motor speed and thus requires sensorymotor integration and a high level of motor processing [19]. It is considered a more complex motor task than others, such as grip strength or finger tapping. As such, it requires more effort and is more sensitive to psychomotor speed [20]. The test has been used extensively for identifying lateralized impairments such as those in Parkinson's disease. In NPH patients, some researchers have used grooved pegboard performance as a psychometric measure for upper extremity motor and psychomotor speed. Upper extremity motor tests may be particularly advantageous in situations where the severity of impairment precludes accurate and reliable assessments of changes in gait.

Herein, we performed a pilot study to investigate changes in grooved pegboard performance before and after CSF TT and its correlation with clinical parameters and white matter lesions and to explore the possibility of its application in combination with the CSF TT.

\section{Methods}

\section{Patients}

Patients $(n=137)$ with physical and cognitive symptoms and brain imaging findings (brain MRI) suggestive of possible NPH were evaluated at Peking Union Medical College Hospital according to the guidelines for the clinical diagnosis of idiopathic NPH (INPH) published in 2005 [21]. The patients had to be older than 40 years of age with insidious progression of symptoms (gait disturbance plus impairments in cognition, bladder control, or both) for at least 3-6 months and have normal CSF opening pressure.

\section{History taking and clinical assessment}

Age, gender, past histories, personal histories, and initial and full-blown symptoms for all patients were recorded. Patients also completed the Mini-Mental State Examination (MMSE) [22], Montreal Cognitive Assessment (MOCA), and activities of daily living (ADL) questionnaire. The iNPH Grading Scale (iNPHGS) [23] was used to rate the severity of each fundamental symptom of iNPH (gait disturbance, cognitive impairment, and urinary incontinence) on a four-point scale after a detailed interview with the patients and caregivers. All subjects underwent a CSF TT and brain MRI.

The CSF TT was performed by lumbar puncture followed by the measurement of CSF opening pressure and CSF collection. We collected $30 \mathrm{ml}$ of CSF or as much as possible until the patients could no longer 
tolerate the procedure. The initial and terminal intracranial pressure was recorded. Before and after the procedure, cognitive function and walking ability were evaluated by the means of the 10-meter walking test and a brief executive function battery, which was previously described [24]. The time and steps that the patient took to complete the 10-meter walking test and a video of the test were recorded. The brief executive function battery included the Symbol-Digit Modalities Test, Trail Making Test A and STROOP-C. Additional evaluations were conducted at 8 hours, 24 hours and 72 hours after CSF TT. In addition to the walking and cognitive function evaluation, the urinary and fecal function questionnaire was completed by the caregiver and patients. The total symptom improvement questionnaire was evaluated by the caregiver and patients at the same time. The following criteria were used to identify responders: 1. CSF TT responders were defined as patients with reductions in their time and number of steps in the walking test at least once after the CSF TT, with decreases of $10 \%$ for both parameters or a decrease of $20 \%$ on at least one of these parameters [25]. 2. Gait was improved based on videos that were evaluated by two neurologists who were blinded to the patient's clinical and neuroimaging characteristics. 3 . The total symptom improvement questionnaire scores were partly or obviously improved. The patients who met 2 of 3 criteria were identified as CSF TT responders.

\section{Brain MRI}

Axial and sagittal spin-echo T1-weighted images were obtained by using a 1.5-T MRI unit (Signa Excite, General Electric, Milwaukee, WI, USA). We also obtained axial fast spin-echo T2-weighted, fluid-attenuated inversion recovery images and diffusion tensor imaging (DTI). The white matter lesions on the axial T2weighted or FLAIR images were scored according to the Fazekas score. The white matter lesions were scored in different regions of the brain, including the periventricular, deep white matter and subcortical white matter lesions. Visual analyses were independently conducted by two neuroradiologists who were experienced in MRI correlates in NPH patients and who were blind to both diagnosis and CSF TT results of the subjects. The fractional anisotropy (FA) and mean diffusivity (MD) values were measured across regions of interest (ROIs) on DTI images. The ROIs were the bilateral anterior and posterior periventricular white matter that were set as circular areas with a diameter of $2 \mathrm{~mm}$ perpendicular to the longitudinal axis of the ipsilateral ventricle on the brain imaging slice with the lateral ventricle. The ROls were chosen according to our pilot study data [26], which indicated that the FA and MD values in the bilateral anterior and posterior periventricular white matter were correlated with walking ability and cognition in possible $\mathrm{NPH}$ patients. A consistency test was performed (Cronbach's a coefficient >0.6).

\section{Statistical methods}

If the data conformed to a normal distribution, the data are presented as the mean \pm standard deviation; if the measurement data conformed to a skewed distribution, the data are presented as the median (interquartile range). The scores for various evaluation indexes before and 4 hours, 8 hours, 24 hours and 72 hours after CSF TT conformed to skewed distributions. The differences in the grooved pegboard test results before and after the CSF TT were analyzed using a nonparametric paired sign rank-sum test. The grooved pegboard test results at the three different time points after the CSF TT (i.e., 8 hours, 24 hours and 72 hours) were also compared by means of a nonparametric paired sign rank-sum test. 
We compared the best improvement ratios for the grooved pegboard test performance between the CSF TT responder and nonresponder groups by means of the Mann-Whitney $U$ test. The improvement ratio was calculated as follows: (baseline performance on the grooved pegboard test - performance after CSF TT) / the baseline performance. The best improvement ratio for the grooved pegboard test was calculated for the different times (i.e., 8 hours, 24 hours, and 72 hours) after CSF TT. The grooved pegboard test and Symbol-Digit Modalities Test performance were combined into the complex visual motor speed index. The improvement ratio for the complex visual motor speed index after CSF TT was also compared between the responder and nonresponder groups using the Mann-Whitney $\mathrm{U}$ test.

The correlations between the grooved pegboard test results and the iNPHGS scores, walking test results, and neuropsychological performance were also tested by means of Spearman correlations. The correlations between the grooved pegboard test results and the DTI parameters from the periventricular white matter lesions were also analyzed using the Spearman correlation method.

There were four time assessments related to the CSF TT, and the Bonferroni correction was applied for the repeated measurements. The statistical significance level was set at $p<0.05 / 4=0.01$. When the Spearman's correlation analysis was used, the statistical significance level was set at $p<0.05$. All statistical analyses were performed with the statistical software package SPSS for Windows (version 13.0.; SPSS Inc., Chicago, IL, USA).

\section{Results}

\section{Demographic characteristics of CSF TT responders and nonresponders}

Seventy-five NPH patients were recruited for the study. There were no significant differences in age, gender, disease duration, or iNPHGS, MMSE, and MOCA scores, but there was a difference with the ADL scores(Table 1). There were 19 patients who underwent shunting operations and were followed up. Among them, 14/19 patients improved after shunting. 
Table 1

Demographic characteristics of the CSF TT responder and nonresponder groups

\begin{tabular}{|llll|}
\hline & Responders $(\mathbf{n}=\mathbf{4 2})$ & Nonresponders $(\mathbf{n}=\mathbf{3 3})$ & P value \\
\hline Age (years old) & $68 \pm 12$ & $68 \pm 11$ & 0.82 \\
\hline Gender (male:female) & $34: 8$ & $26: 7$ & 0.94 \\
\hline Duration (years) & $3.8 \pm 3.3$ & $3.6 \pm 2.8$ & 0.76 \\
\hline iNPHGS total & $5.8 \pm 1.6$ & $5.2 \pm 2.2$ & 0.21 \\
\hline MMSE & $20.5 \pm 7.3$ & $22.9 \pm 6.0$ & 0.23 \\
\hline MOCA & $17.3 \pm 7.1$ & $18.1 \pm 6.6$ & 0.71 \\
\hline ADL & $41.2 \pm 14.5$ & $30.0 \pm 9.5$ & 0.01 * \\
\hline 10-meter walking time (seconds) & $23.4 \pm 28.6$ & $39.2 \pm 130.0$ & 0.45 \\
\hline *P<0.05 & & & \\
\hline
\end{tabular}

\section{The grooved pegboard test performance changes from before to after the CSF TT}

The bilateral grooved pegboard test results at 24 hours and 72 hours after the CSF TT were significantly improved compared with those before the CSF TT $(P<0.01)$ (Table 2). We also tested the grooved pegboard test result differences at 8 hours, 24 hours and 72 hours after the CSF TT. There were significant differences among the three time points with the nondominant hand. With the dominant hand, there were significant differences at 8 hours vs. 72 hours and at 24 hours vs. 72 hours after CSF TT. (Table 2) 
Table 2

Comparison of the grooved pegboard test (GPT) results before and after the CSF TT

\begin{tabular}{|c|c|c|c|c|c|c|}
\hline & $\begin{array}{l}\text { GPT-nondominant } \\
\text { hand }\end{array}$ & Z & $\mathbf{P}$ & $\begin{array}{l}\text { GPT-dominant } \\
\text { hand }\end{array}$ & Z & $\mathbf{P}$ \\
\hline Baseline & $141.0(143)$ & & & $130.2(113.5)$ & & \\
\hline $\begin{array}{l}8 \mathrm{hr} \text { after CSF } \\
\text { TT }\end{array}$ & $134.5(136.5)$ & $-1.63^{1}$ & $0.10^{1}$ & $123.0(90.0)$ & $-2.41^{4}$ & $0.02^{4}$ \\
\hline \multirow[t]{2}{*}{$\begin{array}{l}24 \mathrm{hr} \text { after CSF } \\
\text { TT }\end{array}$} & $131.8(133.4)$ & $-4.21^{1}$ & $0.00^{1 *}$ & $121.4(101)$ & $-3.04^{4}$ & $0.00^{4 *}$ \\
\hline & & $-2.46^{2}$ & $0.01^{2 \star}$ & & $-1.97^{5}$ & $0.05^{5}$ \\
\hline \multirow[t]{3}{*}{$\begin{array}{l}72 \text { hr after CSF } \\
\text { TT }\end{array}$} & $120.3(93.2)$ & $-5.38^{1}$ & $0.00^{1 *}$ & $118.0(92.7)$ & $-3.80^{4}$ & $0.00^{4 *}$ \\
\hline & & $-4.84^{2}$ & $0.00^{2 \star}$ & & $-3.07^{5}$ & $0.00^{5 *}$ \\
\hline & & $-4.40^{3}$ & $0.00^{3 *}$ & & $-2.59^{6}$ & $0.01^{6 *}$ \\
\hline \multicolumn{7}{|l|}{ Note: ${ }^{*} \mathrm{P}<0.01$} \\
\hline \multicolumn{7}{|c|}{${ }^{1}$ compared to the baseline GPT result with the nondominant hand } \\
\hline \multicolumn{7}{|c|}{${ }^{2}$ compared to the GPT result with the nondominant hand at $8 \mathrm{hr}$ after the CSF TT } \\
\hline \multicolumn{7}{|c|}{${ }^{3}$ compared to the GPT result $24 \mathrm{hr}$ after the CSF TT } \\
\hline \multicolumn{7}{|c|}{${ }^{4}$ compared to the baseline GPT result with the dominant hand } \\
\hline \multicolumn{7}{|c|}{${ }^{5}$ compared to the GPT result with the dominant hand $8 \mathrm{hr}$ after the CSF TT } \\
\hline
\end{tabular}

\section{Comparison of the changes in grooved pegboard test results between the CSF TT responders and nonresponders}

The improvement ratio for the grooved pegboard test at 72 hours after the CSF TT was the most significantly improved among the 8-hour, 24-hour, and 72-hour time points after the CSF TT. We compared the improvement ratios for the grooved pegboard test between the CSF TT responder and nonresponder groups at 72 hours after the CSF TT. Performance on the grooved pegboard test combined with the Symbol-Digit Modalities Test was used to produce the complex visual motor speed index. This index for the CSF TT responders and nonresponders was also compared 72 hours after the CSF TT, and there was a significant difference between the two groups $(U=270, P=0.01)$ (Table 3). 
Table 3

The comparison of the improvement ratios for the grooved pegboard test, Symbol-Digit Modalities Test and complex visual motor speed index between the CSF TT responder and nonresponder groups at $72 \mathrm{hr}$ after the CSF TT

\begin{tabular}{|lllll|}
\hline Improvement ratios & $\begin{array}{l}\text { Responders } \\
(\mathbf{n = 4 2})\end{array}$ & $\begin{array}{l}\text { Nonresponders } \\
(\mathbf{n = 3 3})\end{array}$ & $\begin{array}{l}\text { Mann- } \\
\text { Whitney } \\
\mathbf{U}\end{array}$ & $\mathbf{P}$ \\
\hline Grooved pegboard test & $\begin{array}{l}17.8 \\
(23.1) \%\end{array}$ & $10.1(18.8) \%$ & 449 & 0.125 \\
\hline Symbol-Digit Modalities Test & $\begin{array}{l}30.8 \\
(49.6) \%\end{array}$ & $7.7(48.2) \%$ & 310 & 0.03 \\
\hline $\begin{array}{l}\text { Complex visual motor speed index (grooved } \\
\text { pegboard test and Symbol-Digit Modalities }\end{array}$ & $\begin{array}{l}19.2 \\
(30.9) \%\end{array}$ & $9.9(26.1) \%$ & 270 & $0.01^{*}$ \\
\hline Test) & & & & \\
\hline * $<0.01$ & & & & \\
\hline
\end{tabular}

\section{Correlations between grooved pegboard test performance, clinical parameters and periventricular white matter lesion DTI parameters in possible NPH patients}

The baseline grooved pegboard test results correlated significantly with the baseline INPHGS total, INPHGS walking, INPHGS cognition, MMSE, MOCA and ADL scores and 10-meter walking test times ( $P=$ $0.0)$ and did not correlate with the INPHGS urinary score $(P=0.13)$

Twenty-two patients underwent DTI evaluation. The bilateral grooved pegboard test performance correlated with the right periventricular anterior horn FA values (right: $P=0.04$, left: $P=0.02$ ). The right grooved pegboard test results correlated with the left periventricular anterior horn MD values. There were no significant correlations between the grooved pegboard test results and periventricular posterior horn FA and MD values.

\section{Discussion}

The present findings demonstrate that upper extremity motor functions can improve following TT, providing an additional measure of a clinical response. The pegboard test results correlated with cognition and lower extremity motor function. The improvement ratios for the complex visual motor speed index (the grooved pegboard test performance combined with the Symbol-Digit Modalities Test performance) were significantly different between the CSF TT responder and nonresponder groups. Additionally, the results from the pegboard test were correlated with periventricular white matter lesion DTI parameters. 
Upper extremity function is impacted in NPH patients, although lower extremity motor function is the primary concern in NPH patients. There have been several studies on upper extremity motor function after CSF drainage tests and shunting operations in iNPH patients. Tsakanikas D et al reported that these upper extremity motor tests may be useful as sensitive markers of change after shunt placement in iNPH patients (e.g., line tracing tasks). [27] Heather $\mathrm{K}$ et al found significant differences in mental tracking and sustained attention after shunt placement in iNPH patients, consistent with previously reported profiles. [28] Evangelia $L$ et al also found that finger tapping and verbal fluency improvements after CSF TT were useful for the differential diagnosis and prediction of shunt treatment outcomes. [29] The strength of our study was the multiple time point assessments of the upper extremity motor function. The grooved pegboard test showed high sensitivity, and the complex visual motor speed index (a combined measure of grooved pegboard test and Symbol-Digit Modalities Test performance) was more useful in demonstrating significant differences between the CSF TT responder and nonresponder groups. The complex visual motor speed index might be a promising candidate measure to recognize CSF TT responders. Tsakanikas $D$ mentioned that there was no significant decrease in the complex visual motor speed index after CSF TT. [27] However, they performed the evaluation just once, at 2-4 hours after CSF TT, which might have missed the time point showing the best improvement.

Our study also provided evidence on correlations between the grooved pegboard test performance and cognition and walking ability in patients with NPH. The correlation of walking and executive neurocognitive deficits has been reported in NPH patients and supports the notion that gait deficits are due to executive motor planning dysfunction. [30] Upper extremity motor function seems to be consistent with changes in walking ability and cognition, which indicates common mechanisms of motor disturbance and cognitive impairment in NPH patients. This relationship also supported the notion that the grooved pegboard test can be a reliable and useful assessment tool for NPH patients.

The correlation of grooved pegboard test results with the DTI parameters of the periventricular white matter lesions provided mechanistic evidence of upper limb motor dysfunction in NPH patients. White matter lesions are very common in NPH patients. The reduction in the irregular type of periventricular hyperintensity located around the frontal horns after surgery has been reported to be associated with clinical improvements in patients with iNPH. [31] The DTI technique can provide good markers of white matter pathology. Some researchers have reported that brain white matter regions in which FA was decreased after shunt placement were in the corona radiata between the lateral ventricles and the Sylvian fissures in NPH patients. [32] Our study results demonstrated that the grooved pegboard test results correlated with the FA values in white matter lesions on the contralateral anterior periventricular horn. This is consistent with the previous literature and provides more data on the mechanism of motor dysfunction in patients with NPH.

\section{Conclusions}

Our study reported that the performance on the grooved pegboard test was related to lower extremity motor ability and cognitive function. The improvement ratios for the complex visual motor speed index 
(the grooved pegboard test performance combined with the Symbol-Digit Modalities Test performance) were significantly different between the CSF TT responder and nonresponder groups. It might be used as an alternative evaluation tool for patients who are unable to ambulate and may not be able to comply with the gait evaluation.

\section{Limitations}

Our study has several limitations. First, the sample size of patients undergoing surgery was small, and the prediction of the grooved pegboard test was not investigated. Second, this is a pilot study, and the grooved pegboard test results were analyzed among the patients with NPH who completed the multiple time point evaluation after CSF TT. The application of the grooved pegboard test in patients who are unable to ambulate needs to be explored in the future.

\section{Abbreviations}

iNPH: idiopathic normal pressure hydrocephalus; CSF: cerebrospinal fluid; NPH: normal pressure hydrocephalus; MRI: magnetic resonance imaging; CSF TT: cerebrospinal fluid tap test; MMSE: MiniMental State Examination; MOCA: Montreal Cognitive Assessment; ADL: activities of daily living questionnaire; iNPHGS: iNPH Grading Scale; DTI: diffusion tensor imaging; FA: The fractional anisotropy; MD: mean diffusivity; ROls: regions of interest; ml: milliliter.

\section{Declarations}

\section{Authors' contributions}

Caiyan Liu and Jing Gao drafted and revised the manuscript, contributed to the data analysis and interpreted results. Study was designed by Caiyan Liu and Jing Gao. Study supervision and obtaining study funding by Jing Gao and Liying Cui. Caiyan Liu, Liling Dong, Chenhui Mao, Jie Li, Xinying Huang contributed to the clinical assessment and data acquisition. Bo Hou, Feng Feng were responsible to the neuroimaging data collection and analysis. Junji Wei contributed to the neurosurgery treatment for patients. Caiyan Liu performed the statistical analysis. All authors read and approved the final manuscript.

\section{Funding}

This study was supported by a grant from CAMS 2016-12M-1-004, National NSFC81550021 and 2016YFC1306300, and grant XDPB10.

\section{Availability of data and materials}


The anonymized datasets used and/or analyzed during the current study are available from the corresponding author upon reasonable request.

\section{Ethics approval and consent to participate}

The permission for the research was received from the Research Ethics Board of the Peking Union Medical College Hospital (PUMCH). Informed consent was obtained from every patient or a legally authorized representative before undergoing evaluations.

\section{Consent for publication}

Personal information is de-identified in this manuscript.

\section{Competing interests}

The authors declare that they have no competing interests.

\section{References}

1. Adams RD, Fisher CM, Hakim S, Ojemann RG, Sweet WH. Symptomatic occult hydrocephalus with "normal" cerebrospinal-fluid pressure a treatable syndrome. N Engl J Med. 1965;273:117-26.

2. Graff-Radford NR, Jones DT. Normal Pressure Hydrocephalus. Continuum (Minneap Minn). 2019;25(1):165-186. doi: 10.1212/CON.0000000000000689.

3. Isaacs AM, Williams MA, Hamilton MG. Current Update on Treatment Strategies for Idiopathic Normal Pressure Hydrocephalus. Curr Treat Options Neurol. 2019;3;21(12):65. doi: 10.1007/s11940019-0604-z.

4. Meier U, Zeilinger FS, Kintzel D. Signs, symptoms and course of normal pressure hydrocephalus in comparison with cerebral atrophy. Acta Neurochir (Wien). 1999;141(10):1039-48.doi: $10.1007 / \mathrm{s} 007010050480$

5. Palm WM, Saczynski JS, van der Grond J, Sigurdsson S, Kjartansson O, Jonsson PV, et al. Ventricular dilation: association with gait and cognition.Ann Neurol. 2009;66(4):485-93. doi:

10.1002/ana.21739.

6. Blomsterwall E, Bilting M, Stephensen $\mathrm{H}$, Wikkelso $\mathrm{C}$. Gait abnormality is not the only motor disturbance in normal pressure hydrocephalus. Scand J Rehabil Med. 1995;27:205-9.

7. Kang K, Jeon JS, Kim T, Choi D, Ko PW, Hwang SK, et al. Asymmetric and Upper Body Parkinsonism in Patients with Idiopathic Normal-Pressure Hydrocephalus.J Clin Neurol. 2016;12(4):452-459. doi: 10.3988/jcn.2016.12.4.452. 
8. Molde K, Söderström L, Laurell K. Parkinsonian symptoms in normal pressure hydrocephalus: a population-based study.J Neurol. 2017;264(10):2141-2148. doi: 10.1007/s00415-017-8598-5.

9. Mandir AS, Hilfiker J, Thomas G, Minahan RE, Crawford TO, Williams MA, et al. Extrapyramidal signs in normal pressure hydrocephalus: an objective assessment. Cerebrospinal Fluid Res. 2007;13:4-7. doi: 10.1186/1743-8454-4-7

10. Iddon JL, Pickard JD, Cross JJ, Griffiths PD, Czosnyka M, Sahakian BJ. Specific patterns of cognitive impairment in patients with idiopathic normal pressure hydrocephalus and Alzheimer's disease: a pilot study. J Neurol Neurosurg Psychiatry. 1999;67(6):723-32. doi:10.1136/jnnp.67.6.723

11. Klinge P, Ruckert N, Schuhmann M, Dorner L, Brinker T, Samii M. Neuropsychological testing to improve surgical management of patients with chronic hydrocephalus after shunt treatment. Acta Neurochir. Suppl 2002;81:51-3. doi:10.1007/978-3-7091-6738-0_13

12. Kanno S1, Saito M, Hayashi A, Uchiyama M, Hiraoka K, Nishio Y, et al. Counting-backward test for executive function in idiopathic normal pressure hydrocephalus. Acta Neurol Scand. 2012;126(4):279-86. doi: 10.1111/j.1600-0404.2012.01644.x.

13. Damasceno BP, Carelli EF, Honorato DC, Facure JJ. The predictive value of cerebrospinal fluid taptest in normal pressure hydrocephalus. Arq Neuropsiquiatr. 1997;55(2):179-85. doi:10.1590/s0004282x1997000200003

14. Wikkelso C, Andersson H, Blomstrand C, Lindqvist G. The clinical effect of lumbar puncture in normal pressure hydrocephalus. J Neurol Neurosurg Psychiatry. 1982;45(1):64-9. doi:10.1136/jnnp.45.1.64

15. Mihalj M, Dolić K, Kolić K, Ledenko V. CSF tap test - Obsolete or appropriate test for predicting shunt responsiveness? A systemic review. J Neurol Sci. 2016;15(362):78-84. doi: 10.1016/j.jns.2016.01.028.

16. Boon AJ, Tans JT, Delwel EJ, Egeler-Peerdeman SM, Hanlo PW, Wurzer HA, et al. Dutch normalpressure hydrocephalus study: prediction of outcome after shunting by resistance to outflow of cerebrospinal fluid. J Neurosurg. 1997;87(5):687-93. doi:10.3171/jns.1997.87.5.0687

17. Malm J, Kristensen B, Karlsson T, Fagerlund M, Elfverson J, Ekstedt J. The predictive value of cerebrospinal fluid dynamic tests in patients with idiopathic adult hydrocephalus syndrome. Arch Neurol. 1995;52(8):783-9. doi:10.1001/archneur.1995.00540320059013

18. Ravdin LD, Katzen HL, Jackson AE, Tsakanikas D, Assuras S, Relkin NR. Features of gait most responsive to tap test in normal pressure hydrocephalus. Clin Neurol Neurosurg. 2008;110(5):45561. doi:10.1016/j.clineuro.2008.02.003

19. Sage MD, Bryden PJ, Roy EA, Almeida QJ.The relationship between the grooved pegboard test and clinical motor symptom evaluation across the spectrum of Parkinson's disease severity.J Parkinsons Dis. 2012;2(3):207-13. doi: 10.3233/JPD-2012-012093.

20. Mitrushina M1, Fogel T, D'Elia L, Uchiyama C, Satz P. Performance on motor tasks as an indication of increased behavioral asymmetry with advancing age. Neuropsychologia. 1995;33(3):359-64. doi:10.1016/0028-3932(94)00113-4 
21. Marmarou A, Bergsneider M, Relkin N, Klinge P, Black PM. Development of guidelines for idiopathic normal-pressure hydrocephalus: introduction.Neurosurgery. 2005;57(3 Suppl):S1-3; discussion ii-v. doi:10.1227/01.neu.0000168188.25559.0e

22. Folstein MF, Folstein SE, McHugh PR. "Mini-Mental State" a practical method for grading the cognitive state of patients for the clinician. J Psychiatr Res. 1975;12(3):189-98.

23. Kubo Y1, Kazui H, Yoshida T, Kito Y, Kimura N, Tokunaga $H$, et al.Validation of grading scale for evaluating symptoms of idiopathic normal-pressure hydrocephalus. Dement Geriatr Cogn Disord. 2008;25(1):37-45. doi:10.1159/000111149

24. LIU Cai-yan, GAO Jing, MAO Chen-hui, CUI Li-ying, PENG bin, HOU bo, et al. The walking ability and cognitive function changing in normal pressure hydrocephalus patients after cerebrospinal fluid tap test. Zhong Hua Shen Jing Ke Za Zhi. 2016;49(2): 1-5.(in Chinese)

25. Virhammar J1, Cesarini KG, Laurell K.The CSF tap test in normal pressure hydrocephalus: evaluation time, reliability and the influence of pain. Eur J Neurol. 2012;19(2):271-6. doi: 10.1111/j.14681331.2011.03486.x.

26. Caiyan Liu, Liling Dong, Chenhui Mao, Jie Li, Xinying Huang, JunjiWei, et al, Correlation between white matter leisions and clinical features of patients with idiopathic normal pressure hydrocephalus in CSF tap test. Alzheimer's \& Dementia, the journal of Alzheimer's Association.2019;15(7S):711. doi.org/10.1016/j.jalz.2019.06.2732

27. Tsakanikas D, Katzen H, Ravdin LD, Relkin NR. Upper extremity motor measures of Tap Test response in Normal Pressure Hydrocephalus.Clin Neurol Neurosurg. 2009;111(9):752-7. doi: 10.1016/j.clineuro.2009.07.017.

28. Katzen H1, Ravdin LD, Assuras S, Heros R, Kaplitt M, Schwartz TH, et al. Postshunt cognitive and functional improvement in idiopathic normal pressure hydrocephalus. Neurosurgery. 2011;68(2):4169. doi: 10.1227/NEU.0b013e3181ff9d01.

29. Liouta E, Gatzonis S, Kalamatianos T, Kalyvas A, Koutsarnakis C, Liakos F,et al. Finger tapping and verbal fluency post-tap test improvement in INPH: its value in differential diagnosis and shunttreatment outcomes prognosis. Acta Neurochir (Wien). 2017;159(12):2301-2307. doi: 10.1007/s00701-017-3301-2.

30. Beauchet 01, Annweiler C, Montero-Odasso M, Fantino B, Herrmann FR, Allali G.Gait control: a specific subdomain of executive function? J Neuroeng Rehabil. 2012;9(9):12. doi: 10.1186/17430003-9-12.

31. Tullberg M, Jensen C, Ekholm S, Wikkels $\varnothing$ C. Normal pressure hydrocephalus: vascular white matter changes on MR images must not exclude patients from shunt surgery.AJNR Am J Neuroradiol. 2001;22(9):1665-73.

32. Nicot B, Bouzerar R, Gondry-Jouet C, Capel C, Peltier J, Fichten A, et al.Effect of surgery on periventricular white matter in normal pressure hydrocephalus patients: comparison of two methods of DTI analysis. Acta Radiol. 2014;55(5):614-21. doi: 10.1177/0284185113504570. 


\section{Supplementary Files}

This is a list of supplementary files associated with this preprint. Click to download.

- D54SRZ22D4C9BBE8E78447292B1P.pdf 